\title{
Der lange Weg zur personalisierten Medizin in der Allergologie
}

Die Personalisierte Medizin (PM) (auch individualisierte Medizin, stratifizierte Medizin, oder Präzisionsmedizin genannt) stellt eine weitere Evolution des biomedizinischen Fortschrittes dar [1-3]. Das übergeordnete Ziel der personalisierten Medizin besteht darin, das richtige Medikament, in der richtigen Dosis, für den richtigen Patienten, zum richtigen Zeitpunkt einzusetzen. Dabei soll anhand von diversen Biomarkern versucht werden, den komplexen Phänotyp eines Krankheitsbildes in mehr und minder gut definierte Subphänotypen zu zerlegen und die dazu gehörigen Patienten-Untergruppen mit differenzierteren Vorgehensweisen zu diagnostizieren und zu behandeln. Frühzeitige Präventionsmaßnahmen sowie optimierte therapeutische Erfolge sind das oberste Ziel der PM. Mit dem Fortschritt der biomedizinischen Erkenntnisse, insbesondere im Bereich der Genomik, erkennen wir eine zunehmende Tendenz zur Biomarker-basierten Stratifizierung der Patienten, vor allem bei onkologischen $\mathrm{Er}$ krankungen. Theoretisch gilt: Je größer die Heterogenität eines Phänotyps (Krankheitsbild) und je geringer die Erfolge mit einer vermeintlichen Standardtherapie (das sog. „one-size-fits-all“ Modell), desto größer wird sich das Grundprinzip der PM durchsetzen können.

Auf der anderen Seite gibt es wohl kaum ein Krankheitsspektrum, das so heterogen ist wie Allergien. Dennoch neigen wir stets dazu, klassische Phänotypen wie die atopischen Erkrankungen (Asthma, atopische Dermatitis) sehr einheitlich zu betrachten und immer wieder, zum Teil auch durch Leitlinien getrieben, einheitliche Vorgehensweisen in der Behandlung dieser Allergien vorzuschlagen. Als Beispiel sei hier die nunmehr angestrebte Stratifizierung des Asthmas in mehreren Untergruppen auf der Grundlage von validierten Biomarkern genannt. Eine solche Stratifizierung hat bereits zur Entwicklung neuartiger Therapieprinzipien beim Asthma Einzug erhalten und wird in Zukunft zunehmend an Bedeutung gewinnen.
Bei der atopischen Dermatitis (AD) befinden wir uns noch am Anfang einer spannenden Entwicklung. Die AD gilt als Erstmanifestation der atopischen Erkrankungen [4] und stellt vermutlich die Grundlagen für die IgE-vermittelte Sensibilisierung gegen eine Reihe von Allergenen, einschließlich der Nahrungsmittel-, Tierund Umweltallergene. Der Phänotyp ist je nach Alter des Patienten, Stadium der Krankheit (Dauer) oder ethnischem Hintergrund [5] besonders heterogen und spiegelt vermutlich unterschiedliche Pathomechanismen wieder, die eine Dynamik der Immunantwort vermuten lässt $[6,7]$. Nur durch die Auswertung von zahlreichen phänotypischen Informationen aus den Patienten-Registern, idealerweise gekoppelt mit entsprechenden Biobanken, wird es möglich sein, den komplexen Phänotyp der AD anhand von Biomarker-Konstellationen erfolgreich zu stratifizieren und zu einem PM-Ansatz zu führen. Leider kann der PM-basierte Ansatz bei den allermeisten pharmazeutischen Unternehmen außerhalb der onkologischen Indikationen immer noch nicht überzeugen, da diese historisch und Markt-bedingt stets das „one-size-fits-all“ vorziehen.

Allein die Vielfalt der möglichen Allergene als Ursache für die Beschwerden der allergologischen Patienten führt zu einer sehr differenzierten Vorgehensweise sowohl bei der Diagnostik als auch bei der Therapie und bei den möglichen Präventionsmaßnahmen. So ermöglicht die durch die moderne Technologie mit rekombinanten Allergenen bedingte Optimierung der allergologischen Diagnostik auch eine optimierte und personalisierte Immuntherapie allergischer Erkrankungen mit rekombinanten Allergenen.

Fortschritte in den Erkenntnissen der Genetik und der Pathophysiologie allergischer Erkrankungen werden es ermöglichen, eine Vielfalt von wertvollen Biomarkern zu entwickeln, die neben dem diagnostischen Wert darüber hinaus auch als prognostische und prädiktive Biomarker herangezogen werden können. Die damit verbundene Aussicht, Säuglinge mit dem höchsten Risiko zur Entwicklung eines atopischen Marsches frühzeitig zu identifizieren und entsprechende Präventionsmaßnahmen einzuleiten, stellt ein Paradebeispiel von Biomarker-basierter PM dar. Mit der PM und den Möglichkeiten der maßgeschneiderten und gezielten Therapie können mit neuartigen Wirkstoffen sowie Präventionsmaßnahmen auch personalisierte Interventionen im Sinne einer krankheitsverändernden Strategie (Disease modifying strategy) den natürlichen Verlauf dieser Erkrankungen beeinflussen [8]. Derartige Optionen bieten sich nunmehr mit einigen Biologika, die gut definierte immunologische Mechanismen vor allem bei der AD wesentlich spezifischer beeinflussen können und somit das Potenzial aufweisen, langfristige Remissionen hervorzurufen.

\section{Interessenkonflikt}

Prof. Thomas Bieber war in den letzten 5 Jahren bezahlter Referent und/oder Berater für folgende Unternehmen: AbbVie, Allmiral, AnaptysBio, Arena, Asana Biosciences, Astellas, BioVerSys, Boehringer Ingelheim, Celgene, Daiichi-Sankyo, Davos Biosciences, Dermavant/Roivant, DermTreat, DS Pharma, Evaxion, FLX Bio, Galapagos/MorphoSys, Galderma, Glenmark, GSK, Incytes, Kymab, LEO, Lilly, L'Oréal, MenloTx, Novartis, Pfizer, Pierre Fabre, Sanofi/Regeneron, UCB, Vectans.

\section{Autor}

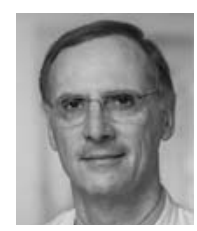

\section{Thomas Bieber}

Prof. Dr. Dr., MDRA, Klinik und Poliklinik für Dermatologie und Allergologie, Universitätsklinikum Bonn

\section{Korrespondenzadresse}

Prof. Dr. Dr. Thomas Bieber, MDRA

Klinik und Poliklinik für Dermatologie und Allergologie

Universitätsklinikum Bonn

Venusberg1

53127 Bonn

Deutschland

Thomas.Bieber@ukbonn.de 


\section{Literatur}

[1] Jameson JL, Longo DL. Precision medicinepersonalized, problematic, and promising. N Engl J Med 2015; 372: 2229-2234

[2] Bieber T. Stratified medicine: A new challenge for academia, industry, regulators and patients. London: Future Medicine; 2013

[3] Bieber T, Vieths S, Broich K. New opportunities and challenges in the assessment of drugs for atopic diseases. Allergy 2016; 71: 1662-1665

[4] Weidinger S, Novak N. Atopic dermatitis. Lancet 2016; 387: 1109-1122

[5] Bieber T. It is time to consider global variations in the clinical phenotype: a commentary on the new diagnostic criteria for atopic dermatitis in children in China. J Eur Acad Dermatol Venereol 2020; 34: 438-439
[6] Bieber T. Novel therapies based on the pathophysiology of atopic dermatitis. J Dtsch Dermatol Ges 2019; 17: 1150-1162

[7] Weidinger S, Beck LA, Bieber T et al. Atopic dermatitis. Nat Rev Dis Primers 2018; 4: 1

[8] Bieber T, Cork M, Reitamo S. Atopic dermatitis: a candidate for disease-modifying strategy. Allergy 2012; 67: 969-975 